\title{
Ligand-free Palladium-Catalyzed Mizoroki-Heck-type Reaction of Arylboronic Acids and Alkenes Using Silver Cation
}

\author{
Eunjin Song, Jinhyung Park, Il-Kwon Oh, ${ }^{\dagger}$ Hyun Min Jung, ${ }^{\dagger}$ and Sunwoo Lee ${ }^{*}$
}

\author{
Department of Chemistry and Institute of Basic Science, Chonnam National University, Gwangju 500-757, Korea \\ *E-mail: sunwoo@chonnam.ac.kr \\ ${ }^{\dagger}$ School of Mechanical Systems Engineering, Chonnam National University, Gwangju 500-757, Korea \\ *Advanced Materials Division, Korea Research Instituted of Chemical Technology, Daejeon 305-600, Korea \\ Received February 26, 2010, Accepted April 5, 2010
}

Key Words: Palladium, Mizoroki-Heck, Arylboronic acid, C-C Bond formation, Silver cation

Transition metal-catalyzed C-C bond formation has been widely used in organic synthesis. ${ }^{1}$ Among this class of reactions, the coupling reaction using organoboronic acid has been intensively studied due to organoboronic acid's advantages of stability to air and moisture, low toxicity and easy availability. ${ }^{2}$ One of the most established reactions is Suzuki cross coupling which is the palladium-catalyzed reaction of arylboronic acid and aryl halides. ${ }^{3,4}$ However, the reaction of arylboronic acid and alkene to afford the Mizoroki-Heck-type product has received less attention, even though this reaction condition is halide free and milder than the original Mizoroki-Heck reaction, which employed aryl halides instead of arylboronic acid. Various new methods have been attempted including a variety of transition metal catalysts $\left(\mathrm{Pd},{ }^{5} \mathrm{Ni}^{6}{ }^{6} \mathrm{Rh},{ }^{7,8}\right.$ and $\left.\mathrm{Ru}^{9}\right)$, base-free condition and microwave irradiation. ${ }^{10}$ When this Mizoroki-Heck-type reaction was carried out with palladium catalyst, nitrogen ${ }^{11,12}$ or phosphine-based ${ }^{13}$ compounds were generally employed as the ligand. Therefore, ligand-free condition capable of showing high catalytic activity and selectivity is required.

It is well established that silver cation can work as an oxidant in the $\mathrm{Pd}(\mathrm{II})$ catalytic system. ${ }^{14}$ However, there are very few examples of the use of silver cations in the palladium-catalyzed Heck-type reaction of arylboronic acid and alkenes. ${ }^{15}$ Here, we report silver cation-mediated Mizoroki-Heck-type reaction to afford the desired product with high yield and high selectivity under ligand-free condition.

We selected a standard Mizoroki-Heck-type reaction, with the phenylboronic acid (1a) and the electron-poor olefin $n$-butyl acrylate (2a), as an appropriate first test reaction. The results are summarized in Table 1 . The coupling reactivity was first investigated with a variety of oxidants or bases. We found that the reactivity and selectivity in favor of the desired product 3 aa was dependent of the nature of the oxidant or base. Among the oxidants and bases screened, AgOAc afforded the desired product in high yield and with high selectivity (entry 1 ). Other silver oxidants such as $\mathrm{Ag}_{2} \mathrm{CO}_{3}$ and $\mathrm{Ag}_{2} \mathrm{O}$ gave low yields (entries 2 and 3), while $\mathrm{Ag}_{2} \mathrm{SO}_{4}$ or benzoquinone, which is one of the most frequently used oxidants, produced very low yields (entries 4 and 5). The reaction using $\mathrm{K}_{3} \mathrm{PO}_{4}$ showed low conversion and afforded a Michael-type addition product as a major one, although at low yield (entry 6). Satoh and Miura reported that $\mathrm{K}_{3} \mathrm{PO}_{4}$ afforded a Michael-type addition product as a major output in the presence of phosphite ligand. ${ }^{16}$ Carbonate bases afforded a trace amount of the desired product (entries 7-9). When phosphite was employed as a ligand, the reactivity and selectivity were not improved (entry 10). Moreover, triphenylphosphine produced $30 \%$ of the byproduct which was derived from the homocoupling of phenylboronic acid (entry 11). All the tested palladium sources showed good yields (entries 1, 12, 13 and 14). A variety of solvents were screened. The addition of water and protic solvents such as $\mathrm{iPrOH}$ and $\mathrm{AcOH}$ showed low product yield, but not Michael-type addition product was found in the reaction mixture (entries 15-18). As the temperature decreased to room temperature, the acrylate was not fully converted (entry 19). When the catalyst loading was decreased to $1 \mathrm{~mol} \%$, the yield was slightly increased to $95 \%$ (entry 20 ). With $0.5 \mathrm{~mol} \% \mathrm{Pd}$ at $110{ }^{\circ} \mathrm{C}$, a $65 \%$ yield was obtained (entry $21)$. The best catalytic system for the Mizoroki-Heck-type reaction, $2 \mathrm{~mol} \% \mathrm{Pd}(\mathrm{OAc})_{2}$ in the presence of $\mathrm{AgOAc}$ with DMF solvent, delivered $95 \%$ yield and full conversion without any trace of byproducts derived from the Michael-type oxidative addition reaction. In addition, we did not detect the byproduct phenol that is derived from the oxidation of phenylboron compound by boron peroxide, which was generated during the catalytic cycle. ${ }^{17}$

With the optimized reaction condition selected from Table 1, the coupled reactions with different arylboronic acids and alkenes are presented in Table 2. The Mizoroki-Heck-type coupling products were obtained as major outputs in all cases. The coupling reactions of phenylboronic acid (1a) with $t$-butyl acrylate (2b), $t$-butyl acrylamide (2c) and styrene (2d) all gave the desired products in high yields (entries 1-3). The para-substituted substrates such as 4-methylphenylboronic acid (1b), 4-ethylphenylboronic acid (1c) and 4-methoxyphenylboronic acid (1d) also afforded the coupled products in moderate to high yields (entries 4-9). The steric demanding substrate 2-methylphenylboronic acid (1e) showed slightly lower yields (55 61\%) (entries 10-12). Attempts to couple with the meta-substituted 2-tolylboronic acid (1f) resulted in reasonable yields (entries 13-15). Among the tested alkene substrates, the coupling reaction of styrene showed yields of over $90 \%$ (entries 3, 7, 12 and 15). However, the coupling reaction of $t$-butyl acrylamide afforded lower product yields of 78, 62, 55 and 65\% (entries 2, 6, 11 and 14). 
Table 1. Optimization of Palladium-catalyzed Mizoroki-Heck-type reaction of phenylboronic acid and $n$-butyl acrylate ${ }^{a}$

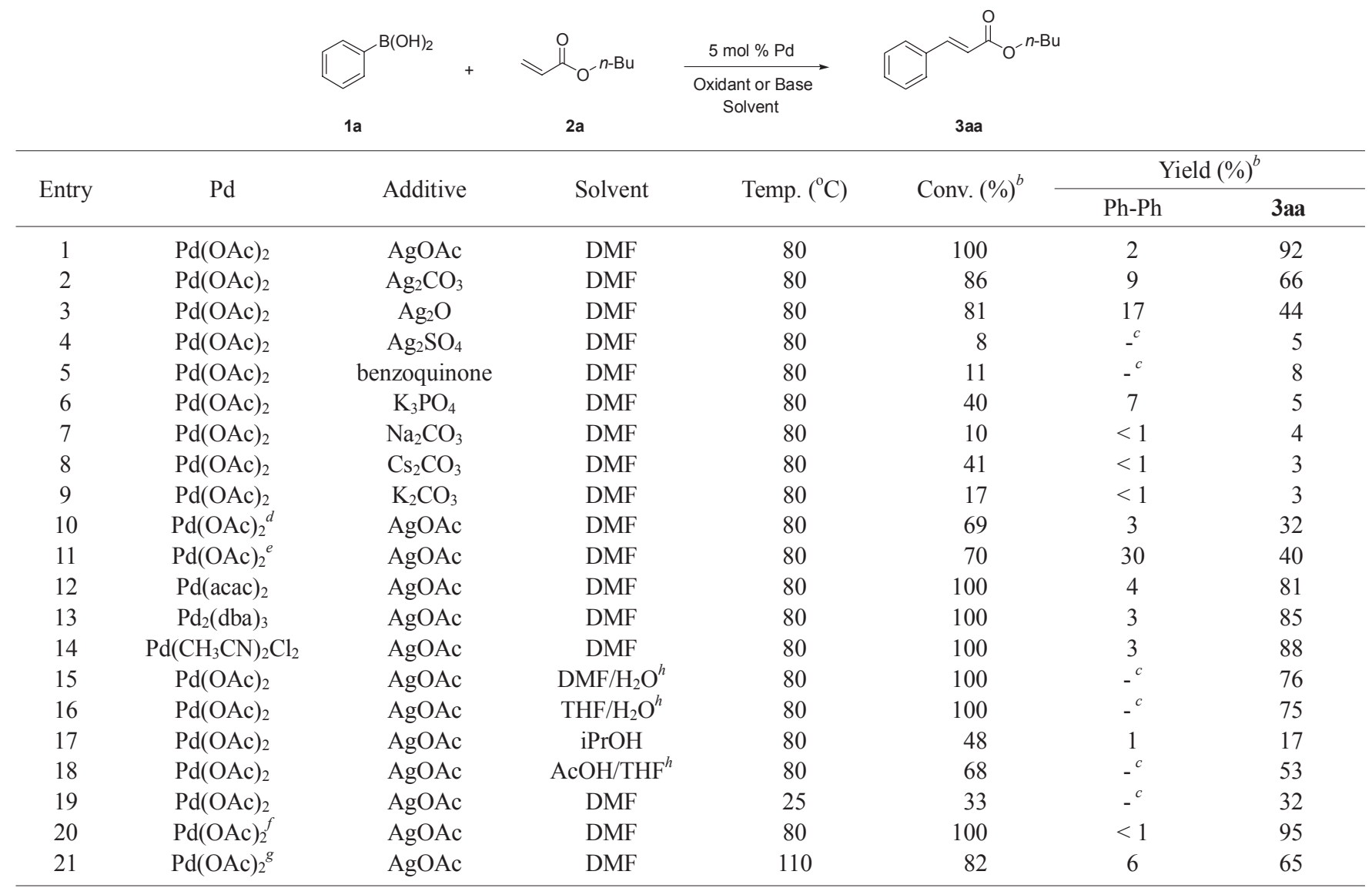

${ }^{a}$ Reaction conditions: 1a $(0.55 \mathrm{mmol}), \mathbf{2 a}(0.5 \mathrm{mmol}), \mathrm{Pd}(0.025 \mathrm{mmol})$, additive $(0.75 \mathrm{mmol})$, and solvent $(2.0 \mathrm{~mL})$ were heated in a sealed tube. ${ }^{b} \mathrm{GC}$ yields were determined by GC by comparison to an internal standard (naphthalene). ${ }^{c}$ Not detected in GC. ${ }^{d}\left[\left(2,4-{ }^{t} \mathrm{Bu}_{2} \mathrm{C}_{6} \mathrm{H}_{3}\right) \mathrm{O}\right]_{3} \mathrm{P}$ was used as a ligand ${ }^{e} \mathrm{PPh}_{3}$ was used as a ligand. ${ }^{f} \mathrm{Pd}(\mathrm{OAc})_{2}(0.01 \mathrm{mmol}),{ }^{g} \mathrm{Pd}(\mathrm{OAc})_{2}(0.005 \mathrm{mmol}){ }^{h}$ The ratio of solvents $=1 / 1(\mathrm{v} / \mathrm{v})$

The chemoselectivity of the system was also briefly explored. Jung's group demonstrated that an amine based-ligand afforded only boron Mizoroki-Heck-type coupled products without any other coupling products. ${ }^{18}$ Brown's group reported that Pd-catalyzed haloboronic acid coupling showed no chemoselectivity in the reaction with $n$-butyl acrylate. ${ }^{9}$ Based on their experiment, we conducted a competition reaction using 4-iodotoluene, phenylboronic acid, and $n$-butyl acrylate under our ligand free catalytic system (Table 3). In contrast to reports from Jung group, the iodo and boron, Mizoroki-Heck coupled product was formed as major and minor output (64\% and 20\% respectively). And Suzuki coupling product was obtained with $4 \%$ yield in the reaction mixture. Instead of 4-iodotoluene, we employed 4-bromotoluene in the competition reaction system. Unlike the case of 4-iodotoluene, only boron Mizoroki-Heck-type coupled product was selectively obtained without any bromo MizorokiHeck and Suzuki coupled products. In the case of the coupling reaction of aryl substrate with butyl acrylate in our system, the order of reactivity was $\mathrm{ArI}>\mathrm{ArB}(\mathrm{OH})_{2}>>\mathrm{ArBr}$.

Our proposed mechanism for the Mizoroki-Heck type reaction involves transmetallation of the arylboronic acid to the palladium(II) complex followed by the insertion of the alkene into the Pd-C bond (Scheme 1). After $\beta$-hydride elimination reaction, the desired Mizoroki-Heck type product was produced, along with $\operatorname{Pd}(0)$ species. Silver cations then oxidized the $\operatorname{Pd}(0)$ to Pd(II) species. ${ }^{19}$ In addition, no Michael-type addition product was obtained in the presence of silver cations, despite the addition of the proton source reagent, such as $\mathrm{PrOH}$ and water, in the reaction mixture (Table 1, entries 15-17). When $\mathrm{PPh}_{3}$ was employed as a ligand in this catalytic system, it promoted the homocoupled byproduct (Table 1, entry 11).

In conclusion, we developed a ligand-free palladium-catalyzed Mizoroki-Heck-type reaction from arylboronic acid and alkene derivatives using silver cations. This simple catalytic system afforded the desired product in high yield and in the competition reaction of 4-bromotoluene, arylboronic acid and

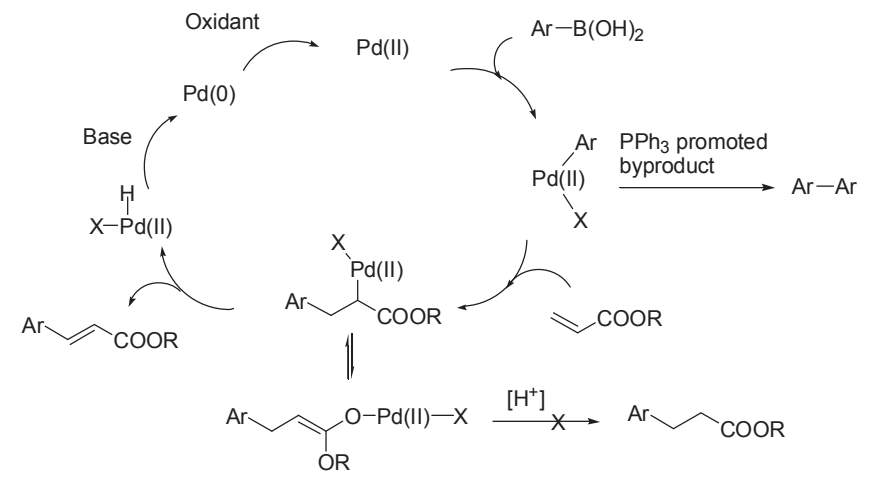

Scheme 1. Proposed mechanism 
Table 2. The Mizoroki-Heck-type reactions of arylboronic acids and alkenes. ${ }^{a}$

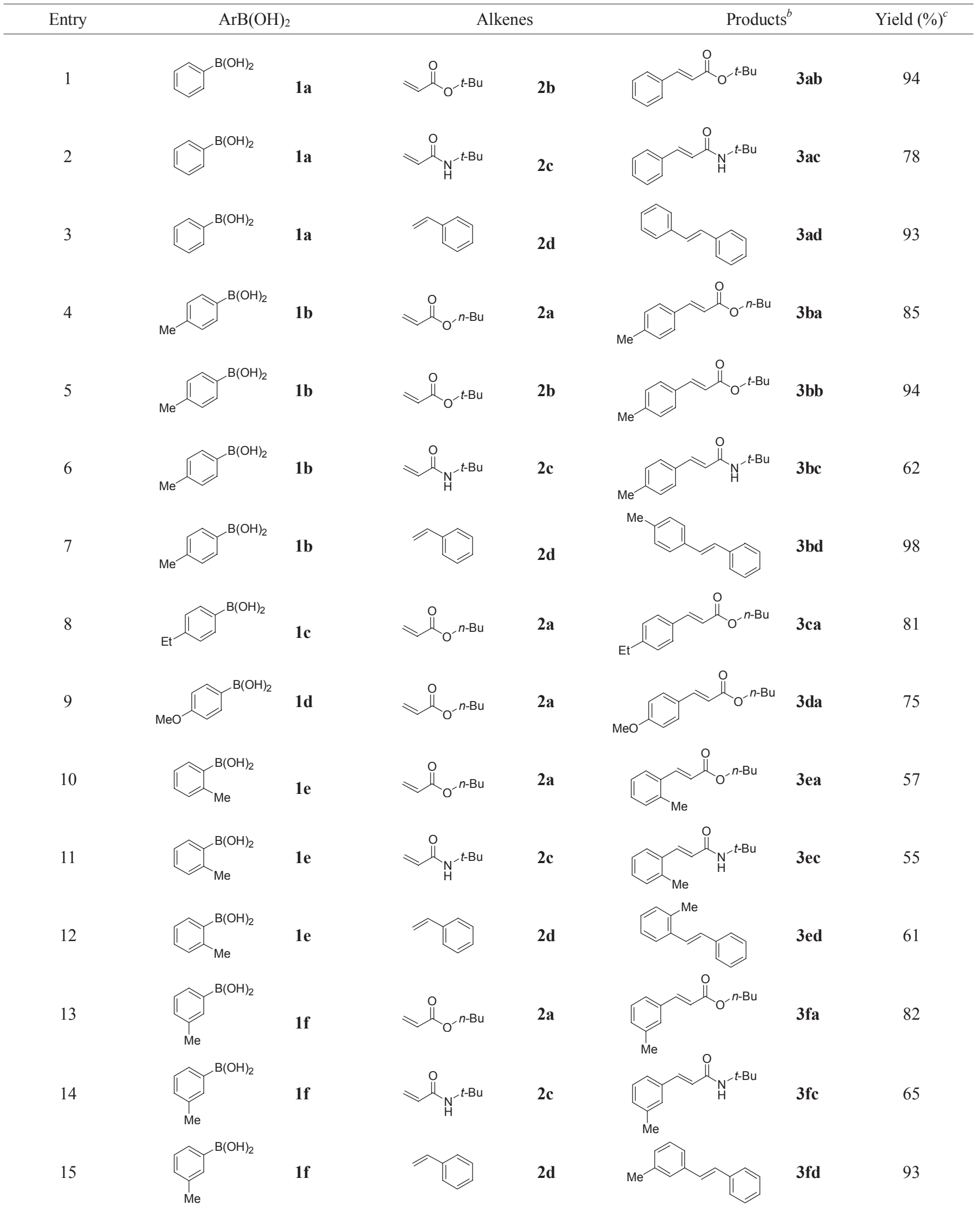

${ }^{a}$ Reaction condition: $\mathrm{ArB}(\mathrm{OH})_{2} 1(3.3 \mathrm{mmol})$, alkene $2(3.0 \mathrm{mmol}), \mathrm{Pd}(\mathrm{OAc})_{2}(0.06 \mathrm{mmol}), \mathrm{AgOAc}(4.5 \mathrm{mmol})$, and DMF $(15.0 \mathrm{~mL})$ were stirred at $80{ }^{\circ} \mathrm{C}$ for $3 \mathrm{~h} .{ }^{b} \mathrm{All}$ compounds were characterized by comparison of gas chromatography $(\mathrm{GC})$ analysis, ${ }^{1} \mathrm{H}$ and ${ }^{13} \mathrm{C}$ NMR spectra with authentic samples or literature data. ${ }^{c}$ Isolated yield. 
Table 3. Competition reactions of $n$-butyl acrylate with phenylboronic acid and aryl halides. ${ }^{a}$

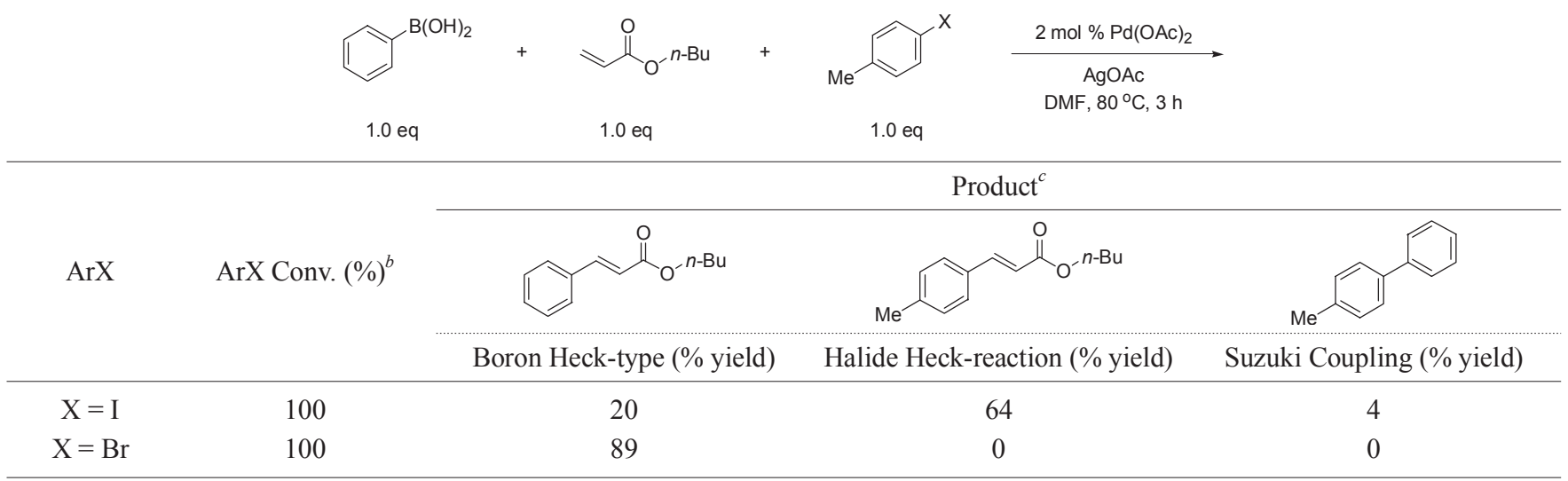

${ }^{a}$ Reaction condition: $\mathrm{PhB}(\mathrm{OH})_{2}(1.0 \mathrm{mmol}), n$-butyl acrylate $(1.0 \mathrm{mmol})$, aryl halide $(1.0 \mathrm{mmol}), \mathrm{Pd}(\mathrm{OAc})_{2}(0.02 \mathrm{mmol})$ and $\mathrm{AgOAc}(1.5 \mathrm{mmol}) \mathrm{were}$ stirred in DMF (5 mL) at $80^{\circ} \mathrm{C}$ for $3 \mathrm{~h} .{ }^{b}$ Conversion yields were determined by gas chromatography (GC) by comparison to an internal standard (naphthalene). ${ }^{c}$ Determined by ${ }^{1} \mathrm{H}$ NMR and GC with internal standard.

n-butyl acrylate, it afforded the boron Mizoroki-Heck-type coupled product exclusively. No homocoupled arylboronic acid or Michael-type byproduct was produced.

\section{General Experimental}

$\mathrm{Pd}(\mathrm{OAc})_{2}(13.5 \mathrm{mg}, 0.06 \mathrm{mmol})$ and phenyl boronic acid (402.4 mg, $3.3 \mathrm{mmol}$ ) and $n$-butyl acrylate $(384.6 \mathrm{mg}, 3.0 \mathrm{mmol})$ were combined with AgOAc (751.1 mg, $4.5 \mathrm{mmol})$ in a small round-bottomed flask. Dimethyl formamide (DMF, $15.0 \mathrm{~mL}$ ) was added and the flask was sealed with a septum. The resulting mixture was placed in an oil bath at $80{ }^{\circ} \mathrm{C}$ for $3 \mathrm{~h}$. The reaction mixture was poured into $30 \mathrm{~mL}$ of $5 \%$ aqueous $\mathrm{LiCl}$ and extracted with $(3 \times 20 \mathrm{~mL}) \mathrm{Et}_{2} \mathrm{O}$. The combined ether extracts were washed with brine $(60 \mathrm{~mL})$, dried over $\mathrm{MgSO}_{4}$, and filtered. The solvent was removed under vacuum, and the resulting crude product was purified by flash chromatography on silica gel. The product was eluted with 5\% ethyl acetate in hexane.

Acknowledgments. This work was supported by the Korea Science and Engineering Foundation (KOSEF) NRL Program grant funded by Korea government (MEST) (No. R0A-2008000-20012-0) and Fundamental R\&D Progarm for Core Technology of Materials of Korean Ministry of Knowledge Economy.

\section{References}

1. de Meijer, A.; Diederich, F. Metal-Catalyzed Cross-Coupling
Reactions, 2nd ed.; Vol. 1 and 2, Wiley-VCH: 2004.

2. Hall, D. G. Boronic Acids; Wiley-VCH: 2005.

3. Miyaura, N.; Suzuki, A. Chem. Rev. 1995, 95, 2457.

4. Nakamura, I.; Yamamoto, Y. Chem. Rev. 2004, 104, 2127.

5. Nishikata, T.; Yamamoto, Y.; Miyaura, M. Angew. Chem. Int. Ed. 2003, 42, 2768.

6. Lin, P.-S.; Jeganmohan, M.; Cheng, C.-H. Chem. Asian J. 2007, $2,1409$.

7. Zou, G.; Wang, Z.; Zhu, J.; Tang, J. Chem. Commun. 2003, 2438.

8. Martinez, R.; Voica, F.; Genet, J.-P.; Darses, S. Org. Lett. 2007, 9 , 3213.

9. Farrington, E. J.; Barnard, C. F. J.; Rowsell, E.; Brown, J. M. Adv. Synth. Catal. 2005, 347, 185.

10. Lindh, J.; Enquist, P.-A.; Pilotti, A.; Nilsson, P.; Larhed, M. J. Org. Chem. 2007, 72, 7957.

11. Enquist, P.-A.; Lindh, J.; Nilsson, P.; Larhed, M. Green Chem. 2006, 8, 338 .

12. Gupta, A. K.; Song, C. H.; Oh, C. H. Tetrahedron Lett. 2004, 45 , 4113.

13. Ruan, J.; Li, X.; Saidi, O.; Xiao, J. J. Am. Chem. Soc. 2008, 130, 2424.

14. Beccalli, E. M.; Broggini, G.; Martinelli, M.; Sottocornola, S. Chem. Rev. 2007, 107, 5318.

15. Su, Y.; Jiao, N. Org. Lett. 2009, 11, 2980.

16. Horiguchi, H.; Tsurugi, H.; Satoh, T.; Miura, M. J. Org. Chem. 2008, 73, 1590.

17. Jung, Y. C.; Mishra, R. K.; Yoon, C. H.; Jung, K. W. Org. Lett. 2003 , 5,2231 .

18. Yoo, K. S.; Yoon, C. H.; Jung, K. W. J. Am. Chem. Soc. 2006, 128 , 16384.

19. Du, X.; Suguro, M.; Hirabayashi, K.; Mori, A.; Nishikata, T.; Hagiwara, N.; Kawata, K.; Okeda, T.; Wang, H. F.; Fugami, K.; Kosugi, M. Org. Lett. 2001, 3, 3313. 